\title{
Platelet count is closely associated with the severity of liver injury in patients with chronic hepatitis $B$ virus infection: A cross-sectional study
}

\author{
YA-TING YANG $^{1,2^{*}}$, LI-LI WANG ${ }^{3 *}$, LI-TING YAN $^{1,2}$, LI-TING ZHANG $^{1}$, WEI ZHOU ${ }^{2}$, \\ QING-FENG CHEN ${ }^{1}$, YU CHEN ${ }^{4}$, SU-JUN ZHENG ${ }^{4}$, ZHONG-PING DUAN $^{4}$ and JUN-FENG LI ${ }^{1,2}$ \\ ${ }^{1}$ Department of Infectious Diseases; ${ }^{2}$ Institute of Infectious Diseases; ${ }^{3}$ Department of Radiology, \\ The First Hospital of Lanzhou University, Lanzhou, Gansu 730000; ${ }^{4}$ Artificial Liver Center, \\ Beijing YouAn Hospital, Capital Medical University, Beijing 100000, P.R. China
}

Received September 18, 2019; Accepted January 16, 2020

DOI: $10.3892 /$ etm.2020.8703

\begin{abstract}
Although the platelet count may provide clues regarding the severity of liver disease, there are currently no available data supporting the utility of the platelet count to evaluate the degree of liver injury in patients with chronic hepatitis B virus (HBV) infection. The present study aimed to determine the association between the platelet count and the severity of liver injury in patients with chronic HBV infection. A total of 941 patients were included and were stratified into a Child-Turcotte-Pugh (CTP) class A group and a CTP class B/C group using the CTP scoring system. A total of 53 patients underwent liver biopsy. The pathological stage F4 was defined as cirrhosis based on the METAVIR scoring system. Compared with that in patients with CTP class A, the platelet count in patients with CTP class B/C was lower $(\mathrm{P}<0.001)$. Similarly, for patients with normal alanine aminotransferase (ALT) levels, the platelet count was significantly different between the CTP class $\mathrm{B} / \mathrm{C}$ and $\mathrm{A}$ groups $(\mathrm{P}<0.001)$. The platelet count was inversely correlated with the CTP score $(\mathrm{r}=-0.420, \mathrm{P}<0.001)$ and independently associated with CTP grade B/C [odds ratio (OR), 0.994; 95\% CI, 0.990-0.999; $\mathrm{P}=0.009]$. The area under the receiver operating characteristic curve (AUC) of the platelet count to distinguish CTP grade $\mathrm{B} / \mathrm{C}$ from $\mathrm{A}$ was 0.712 and 0.791 , respectively, in all patients with HBV infection and the subset with normal ALT levels. In addition, compared to patients with chronic hepatitis B, patients with cirrhosis had a lower platelet count and higher
\end{abstract}

Correspondence to: Dr Jun-Feng Li, Department of Infectious Diseases, The First Hospital of Lanzhou University, 1 Donggangxi Road, Lanzhou, Gansu 730000, P.R. China

E-mail: junfenglee@126.com

${ }^{*}$ Contributed equally

Key words: platelet count, liver injury, liver fibrosis, chronic hepatitis B virus infection aspartate transaminase-to-platelet ratio index (APRI) and fibrosis index based on four factors (FIB-4) $(\mathrm{P}<0.001)$. The platelet count was inversely correlated with FIB-4 ( $r=-0.855$, $\mathrm{P}<0.001)$ and APRI $(\mathrm{r}=-0.741, \mathrm{P}<0.001)$. The AUC for the platelet count to distinguish cirrhosis from chronic hepatitis B was 0.927 (sensitivity, 78.76\%; specificity, 92.22\%). Among patients who underwent liver biopsy, the platelet count in those with F4 was lower compared with that in patients with $\leq \mathrm{F} 3$ $(\mathrm{P}=0.013)$. The platelet count was inversely correlated with the pathological stage $(\mathrm{r}=-0.295, \mathrm{P}=0.032)$ and was independently associated with F4 (OR, 0.978; 95\% CI, 0.960-0.997; $\mathrm{P}=0.026$ ). The AUC of the platelet count to distinguish $\mathrm{F} 4$ from patients with $\leq \mathrm{F} 3$ was 0.761 . In conclusion, the platelet count may be used as a non-invasive marker to assess the severity of liver injury and of liver fibrosis in patients with chronic HBV infection.

\section{Introduction}

Chronic hepatitis $\mathrm{B}(\mathrm{CHB})$ is a major public health concern with a global prevalence. Approximately 65,000 patients die of liver failure, liver cirrhosis and hepatocellular carcinoma caused by hepatitis B virus (HBV) infection each year (1). Significant liver fibrosis and cirrhosis cause severe liver injury in patients with $\mathrm{CHB}$ and are important indicators for anti-viral therapy in those patients. At present, liver biopsy is considered the gold standard for staging of the degree of liver injury, but it is not widely used due to its invasiveness and risk of complications. Even if several non-invasive tests are applied for evaluating liver injury, in general, the early diagnosis of cirrhosis still depends on liver biopsy, as these non-invasive indicators are currently not ideal.

Platelets contain numerous growth factors that are required for organ development, tissue regeneration and repair $(2,3)$. It was reported that in chronic liver diseases, platelets participate not only in liver injury through $\mathrm{T}$ cell-mediated immune responses but also in tissue repair through secretion of growth factors $(4,5)$. Although thrombocytopenia is one of the common complications in patients with chronic liver disease in the clinic, there is no available data supporting that the degree of liver injury in patients 
with chronic HBV infection or cirrhosis associated with HBV may be evaluated based on the platelet count. Only several studies have suggested that the etiology of thrombocytopenia in liver disease may be attributed to splenomegaly, hypersplenism, portal hypertension and decreased thrombopoietin production $(6,7)$. A recent study reported that in patients with $\mathrm{CHB}$ with significant fibrosis receiving anti-viral therapy, the increase of platelets was associated with the improvement of liver fibrosis (8). For hepatitis C, a clinical study has suggested that the platelet count may predict significant hepatitis C-associated fibrosis (9), and recently, the platelet count was reported to be able to indicate the degree of liver steatosis and fibrosis $(10,11)$, and may serve as a clue regarding the severity of disease in patients with non-alcoholic fatty liver disease (12).

Overall, the association between thrombocytopenia and hepatic pathogenesis remains obscure and the role of platelets in chronic liver disease has remained largely elusive. There are currently no available data to support that the platelet count is able to evaluate the severity of liver injury in patients with chronic HBV infection. Using the results of liver biopsy as the gold standard, the present cross-sectional study aimed to evaluate the role of the platelet count in estimating liver injury in patients with chronic HBV infection.

\section{Materials and methods}

Patients. In the present study, 1,597 patients with chronic HBV infection at the First Hospital of Lanzhou University (Lanzhou, China), who met the inclusion criterion of positive serum HBV surface antigen (HBsAg) for $>6$ months, were enrolled between June 2016 and August 2017. The exclusion criteria were as follows: HBsAg-negative status; age $<18$ years; co-infection with hepatitis $\mathrm{A}, \mathrm{C}$ and/or $\mathrm{D}$ virus; autoimmune hepatitis; alcoholic liver disease; non-alcoholic fatty liver disease; Wilson's disease; primary biliary cholangitis; drug-induced liver injury; co-existent hematologic diseases; history of splenectomy; history of therapy with anti-platelet drugs, immune inhibitors, molecular targeted drugs and hepatotoxic drugs; incomplete data; liver transplantation; extrahepatic tumor; multiple organ failure and obstructive jaundice. In total, 941 patients were finally included, of whom 347 and 226 patients were diagnosed with cirrhosis and $\mathrm{CHB}$, respectively, based on the diagnostic criteria $(1,13)$. Diagnostic data were obtained from clinical, imaging examination or liver biopsy results. Cirrhosis was classified as the compensated $(\mathrm{n}=170)$ or decompensated stage $(\mathrm{n}=177)$. This classification depends on the presence or absence of clinically evident decompensating events (specifically ascites, variceal hemorrhage and encephalopathy) (13). A flow chart of the patients included in the study is provided in Fig. 1.

This study was approved by the Ethics Committee of the First Hospital of Lanzhou University (Lanzhou, China; no. LDYYLL2019-209).

Laboratory tests and imaging examination. All patients included had complete biochemical, routine blood and coagulation test results, which were respectively detected by an automatic biochemical analyzer (AU400; Olympos Corp.), an automatic blood cell analyzer (BC-5390CRP; Mindray) and an automatic coagulation Analyzer (Precil C3510; Beijing Precil Instrument Co., Ltd.). The serum levels of alanine aminotransferase (ALT) and aspartate aminotransferase (AST) >40 IU/1 were considered abnormal. Splenic thickness, portal vein diameter and portal vein velocity were measured on an Ultrasound Machine (GE Logiq E9; GE Healthcare) by an experienced ultrasonographist.

Pathological assessment and indices calculated. Liver pathological assessment was based on the METAVIR scoring system (14). In the present study, F4 was defined as cirrhosis. The formulas for calculating the platelet count to splenic thickness ratio (PC/ST ratio), fibrosis index based on four factors (FIB-4) and AST-to-platelet ratio index (APRI) were as follows $(15,16)$ : Platelets/splenic thickness (PC/ST) ratio=platelet count $\left(10^{\circ} / \mathrm{l}\right) /$ splenic thickness $(\mathrm{mm})$; APRI=AST (IU/l)/its upper limit of normal (ULN)/platelet count $\left(10^{9} / 1\right)$ $\mathrm{x} 100$. The ULN for AST was $40 \mathrm{IU} / \mathrm{l}$. FIB-4=age (years) $\mathrm{x}$ AST/platelet count x [ALT (IU/l) $]^{1 / 2}$.

Statistical analysis. Epidata Software (version 3.1; Epidata) was used to manage data. SPSS software (version 17.0; SPSS, Inc.) was used to perform statistical analysis. Continuous variables are expressed as the mean \pm standard deviation and categorical variables as $\mathrm{n}(\%)$. One-sample Kolmogorov-Smirnov test was applied to determine if a set of data was normally distributed. For intergroup comparisons of continuous variables, variables with a normal and non-normal distribution were assessed by the independent-samples t-test and the non-parametric U Mann-Whitney test, respectively. The $\chi^{2}$ test was used for categorical variables. To assess the association between variables and the Child-Turcotte-Pugh (CTP) score (17) or pathological stage, Spearman's rank correlation coefficient was determined. Multivariate logistic regression analysis was performed to identify the independent factors predicting liver function impairment. Finally, receiver operating characteristic (ROC) curve analysis was used to evaluate the diagnostic performance of the platelet count. $\mathrm{P}<0.05$ was considered to indicate statistical significance.

\section{Results}

Patient characteristics. The present study included 941 patients with a mean age of $50.29 \pm 10.93$ years (range, 18-90 years). The study population comprised 657 (69.82\%) male patients and $284(30.18 \%)$ female patients. These patients were stratified into a CTP class A group and a CTP class B/C group using the CTP scoring system. The characteristics of the patients included are summarized in Table SI. Compared with those of CTP class A, those with CTP class B/C had a lower age $(\mathrm{P}=0.009)$, platelet count $(\mathrm{P}<0.001)$, albumin $(\mathrm{P}<0.001)$ and prothrombin activity $(\mathrm{PTA} ; \mathrm{P}<0.001)$, and had a greater splenic thickness $(\mathrm{P}<0.001)$ and portal vein diameter $(\mathrm{P}<0.001)$. Similarly, in patients with normal ALT levels, age, platelet count, albumin and PTA were significantly lower, whilst splenic thickness and portal vein diameter were significantly higher in patients with CTP class B/C vs. A (Table I).

Platelet count is associated with the severity of liver function in patients with chronic HBV infection. In terms of liver function, correlation analysis revealed that the CTP score was inversely correlated with the platelet count, albumin, 


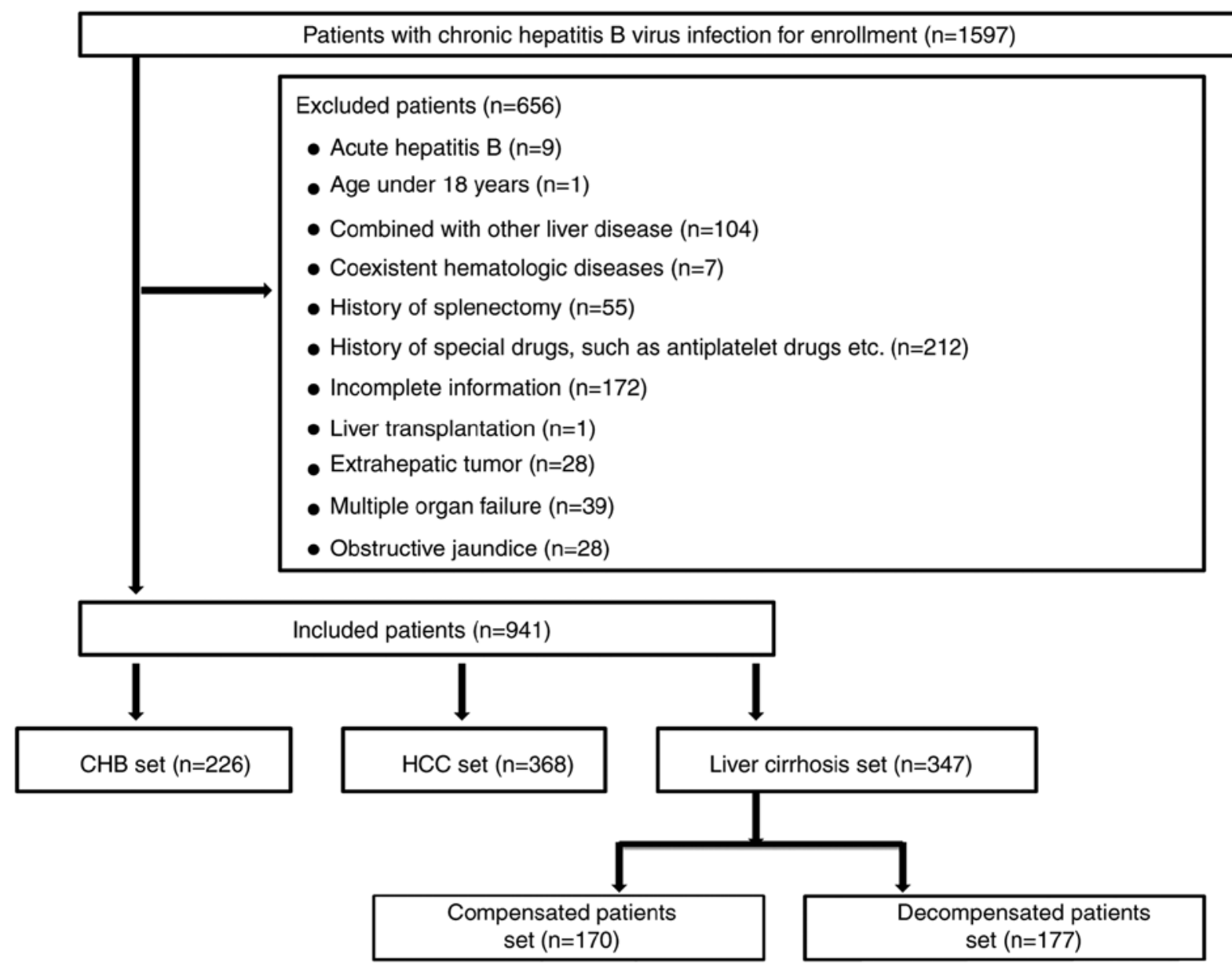

Figure 1. Flow chart of patients in the study. CHB, chronic hepatitis B; HCC, hepatocellular carcinoma.

cholinesterase (CHE) and PTA (Table SII). In addition, the platelet count correlated positively with albumin $(r=0.350$, $\mathrm{P}<0.001)$, CHE $(\mathrm{r}=0.500, \mathrm{P}<0.001)$ and PTA $(\mathrm{r}=0.503$, $\mathrm{P}<0.001)$, and correlated inversely with prothrombin time (PT; $\mathrm{r}=-0.522, \mathrm{P}<0.001$; Table SIII). Multivariate logistic regression analysis verified that the platelet count [odds ratio (OR), 0.994; $\mathrm{P}=0.009$ ], albumin (OR, 0.703; $\mathrm{P}<0.001)$, total bilirubin $(\mathrm{OR}, 1.146 ; \mathrm{P}<0.001)$ and age $(\mathrm{OR}, 0.971 ; \mathrm{P}=0.010)$ were independently associated with CTP class B/C (Table SIV). Furthermore, in patients with normal ALT levels, the platelet count, albumin and PTA were also independently associated with CTP class B/C (Table II). ROC curve analysis indicated that the area under the curve (AUC) of the platelet count to distinguish CTP class B/C from A were 0.712 (Fig. 2A) and 0.791 (Fig. 2B) in patients with chronic HBV infection and normal ALT levels, respectively.

The platelet count is able to identify patients with cirrhosis among those with chronic $H B V$ infection. Among the patients with chronic HBV infection, those with cirrhosis had a significantly lower age, platelet count, albumin and PTA, and significantly higher PT, splenic thickness, portal vein diameter, CTP score, APRI and FIB-4 than those with no cirrhosis (Table SV). Correlation analysis demonstrated that the platelet count was inversely correlated with FIB-4 $(\mathrm{r}=-0.855, \mathrm{P}<0.001)$ and APRI $(\mathrm{r}=-0.741, \mathrm{P}<0.001)$, whereas splenic thickness and CTP scores correlated positively with FIB-4 (Table SVI). These three indicators and age were then included in a multivariate analysis. The results suggested that the platelet count, CTP score and splenic thickness were independently associated with cirrhosis (Table SVII). The ROC curve analysis indicated that the AUC of the platelet count for cirrhosis was $0.927(\mathrm{P}<0.001)$, the AUC of splenic thickness for cirrhosis was $0.903(\mathrm{P}<0.001)$, the AUC of CTP score for cirrhosis was $0.838(\mathrm{P}<0.001$; Fig. $3 \mathrm{~A}$ and Table SVIII).

The platelet count has potential diagnostic value for early-stage cirrhosis. The stage of $\mathrm{CHB}$ and compensated cirrhosis are indistinguishable if no invasive examination is performed. In the present study, patients with compensated cirrhosis, compared to those with $\mathrm{CHB}$, also had a lower age, platelet count and albumin, and higher splenic thickness, APRI, FIB-4 and CTP score $(\mathrm{P}<0.001)$. The differences in these groups are provided in the supplementary material (Table SIX). Logistic regression analysis indicated that the platelet count was also independently correlated with compensated cirrhosis (OR, 0.966; P<0.001; Table SX). The AUC of the platelet count for compensated cirrhosis was 0.912 and the optimal cut-off value was $117 \times 10^{9} / 1$, with a sensitivity of $80.09 \%$ and a specificity of $90.00 \%$ (Fig. 3B). 
Table I. Baseline characteristics of the study population of patients of chronic hepatitis B virus infection with normal ALT.

\begin{tabular}{lccr}
\hline Variable & CTP class A $(\mathrm{n}=395)$ & CTP class B/C (n=137) & P-value \\
\hline Age $($ years $)$ & $51.61 \pm 11.19$ & $49.22 \pm 11.59$ & 0.028 \\
Male sex & $250(63.29)$ & $84(61.31)$ & 0.757 \\
Platelet count $\left(\mathrm{x} 10^{9} / \mathrm{l}\right)$ & $124.76 \pm 67.60$ & $64.18 \pm 44.70$ & $<0.001$ \\
PC/ST ratio & $172,2.80 \pm 2.15$ & $92,1.12 \pm 0.94$ & $<0.001$ \\
AST $(\mathrm{U} / \mathrm{l})$ & $33.21 \pm 16.77$ & $49.68 \pm 49.11$ & $<0.001$ \\
TP $(\mathrm{g} / \mathrm{l})$ & $69.91 \pm 7.56$ & $62.08 \pm 10.20$ & $<0.001$ \\
Albumin $(\mathrm{g} / \mathrm{l})$ & $43.24 \pm 4.28$ & $34.30 \pm 6.20$ & $<0.001$ \\
TBIL $(\mu$ mol/l) & $20.10 \pm 8.64$ & $46.54 \pm 34.41$ & $<0.001$ \\
DBIL $(\mu$ mol/l) & $4.66 \pm 2.15$ & $15.09 \pm 15.48$ & $<0.001$ \\
CHE $(\mathrm{KU} / \mathrm{l})$ & $6.19 \pm 1.88$ & $3.28 \pm 1.56$ & $<0.001$ \\
TC $(\mathrm{mmol} / \mathrm{l})$ & $3.78 \pm 0.92$ & $2.55 \pm 0.95$ & $<0.001$ \\
PT $(\mathrm{sec})$ & $12.41 \pm 1.17$ & $16.42 \pm 4.38$ & $<0.001$ \\
PTA $(\%)$ & $86.32 \pm 14.57$ & $59.29 \pm 14.67$ & $<0.001$ \\
APTT $(\mathrm{sec})$ & $33.86 \pm 5.58$ & $38.67 \pm 15.65$ & $<0.001$ \\
Splenic thickness $(\mathrm{n}, \mathrm{mm})$ & $172,45.09 \pm 11.82$ & $92,57.49 \pm 10.78$ & $<0.001$ \\
Portal vein diameter $(\mathrm{n}, \mathrm{mm})$ & $211,11.71 \pm 1.95$ & $90,13.48 \pm 2.30$ & $<0.001$ \\
FIB-4 & $4.14 \pm 4.75$ & $9.63 \pm 7.43$ & $<0.001$ \\
APRI & $1.02 \pm 1.31$ & $2.56 \pm 2.00$ & $<0.001$ \\
& & &
\end{tabular}

Values are expressed as the mean \pm standard deviation or $\mathrm{n}(\%)$. CTP, Child-Turcotte-Pugh; PC/ST ratio, platelet count to splenic thickness ratio; AST, aspartate aminotransferase; TBIL, total bilirubin; DBIL, direct bilirubin; IBIL, indirect bilirubin; TP, total protein; CHE, cholinesterase; TC, total cholesterol; PT, prothrombin time; PTA, prothrombin activity; APTT, activated partial thromboplastin time; FIB-4, fibrosis index based on four factors; APRI, AST-to-platelet ratio index.

Table II. Independently associated indicators of moderate to severe liver function impairment in patients with normal alanine aminotransferase obtained by multivariate analysis of the indicators with statistical differences in Table I.

\begin{tabular}{lccr}
\hline Variable & OR & $95 \%$ CI & P-value \\
\hline Platelet count & 0.991 & $0.985-0.998$ & 0.007 \\
Albumin & 0.773 & $0.719-0.831$ & $<0.001$ \\
PTA & 0.902 & $0.875-0.931$ & $<0.001$ \\
Age & 0.973 & $0.946-1.002$ & 0.067 \\
\hline
\end{tabular}

OR, odds ratio; CI, confidence interval; PTA, prothrombin activity.

Platelet count is closely associated with histological severity in patients with chronic HBV infection. A total of 53 patients included underwent liver biopsy and were stratified into the $\leq F 3$ set $(n=6)$ and $F 4$ set $(n=47)$ according to the METAVIR scoring system. The study population with a mean age of $50.15 \pm 8.55$ years comprised $40(75.47 \%)$ male patients and $13(24.53 \%)$ female patients. The baseline characteristics of these patients are provided in Table III. The patients with F4 had a significantly lower platelet count, albumin, $\mathrm{CHE}$ and PTA $(\mathrm{P}<0.05)$, and correlation analysis suggested that these parameters were significantly negatively correlated with all pathological stages (Table IV). The multivariate logistic regression analysis revealed that only the platelet count (OR, 0.978;
$\mathrm{P}=0.026$ ) was independently associated with F4 (Table V). The AUC of the platelet count to distinguish $\mathrm{F} 4$ from $\leq \mathrm{F} 3$ was 0.761 with a sensitivity of $66.7 \%$ and specificity of $80.9 \%$ (Fig. 4).

\section{Discussion}

Several clinical studies indicated that the platelet count is able to distinguish significant fibrosis in patients with hepatitis $\mathrm{C}$ infection and may be used to assess the degree of liver steatosis and fibrosis in patients with non-alcoholic fatty liver disease $(11,18)$. Furthermore, it has been indicated that the platelet count is able to modulate the quantitative association between HBV DNA and surface antigen concentrations (19), and HBsAg levels were able to predict the degree of fibrosis in patients with CHB (20). Therefore, the platelet count is closely linked to the HBV infection status. Furthermore, a study reported that the platelet count was associated with inflammation and fibrosis of the liver in patients with chronic HBV infection (21). However, at present, only a small amount of evidence is available to support the association of the platelet count with the severity of liver injury in patients with chronic HBV infection. In the present study, a cohort of patients with chronic HBV infection that met the study requirements based on rigorous criteria were ultimately included. Based on the above standards and the liver biopsy data, the ability of the platelet count to evaluate chronic liver injury in patients with HBV infection was confirmed. The results suggested that the platelet count was closely linked to the severity of liver injury in patients with chronic HBV infection. 

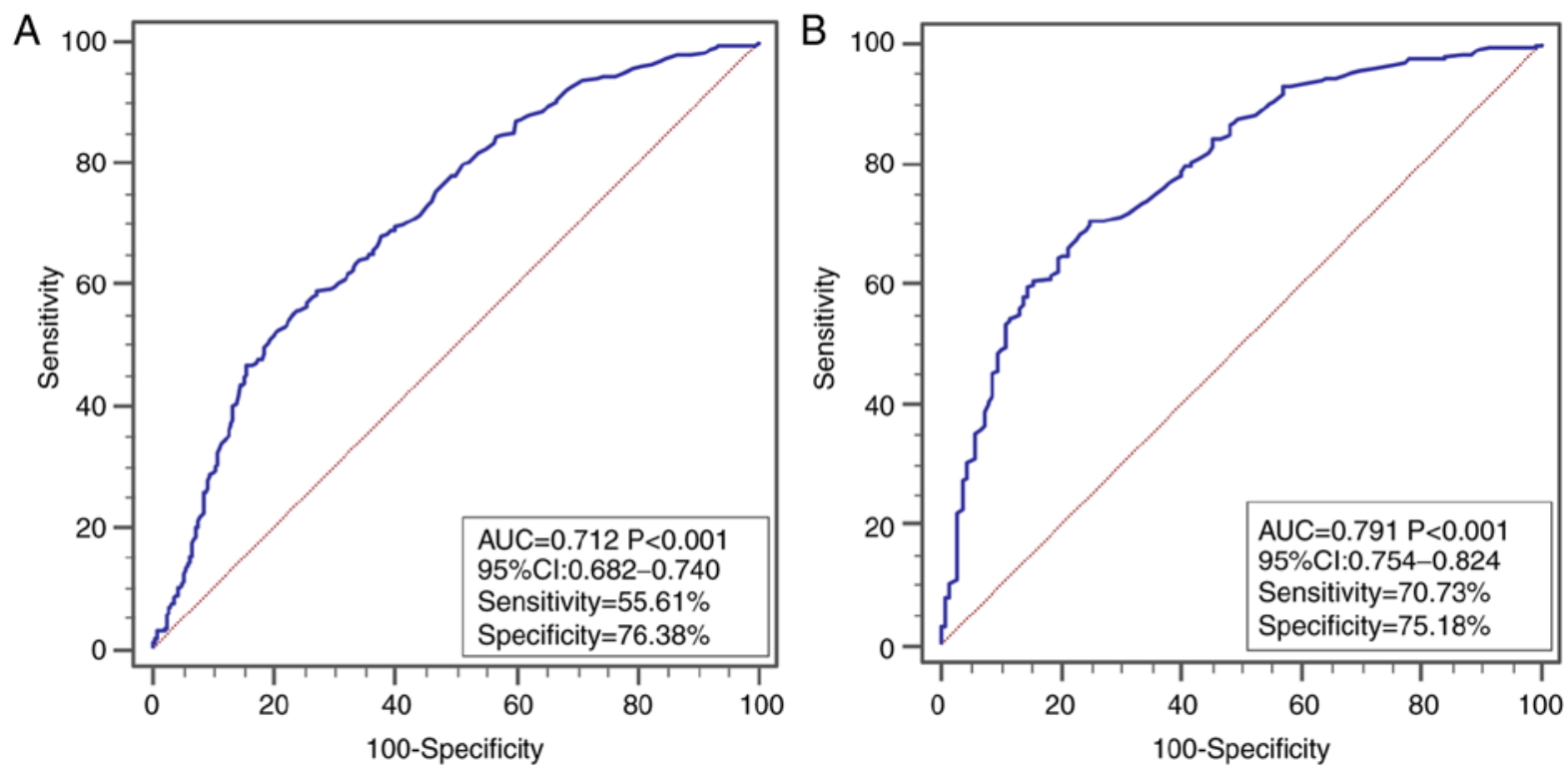

Figure 2. The ROC of platelet count for moderate to severe liver function impairment in (A) patients with chronic HBV infection and (B) patients with chronic $\mathrm{HBV}$ infection and normal alanine aminotransferase levels. AUC, area under the ROC curve; ROC, receiver operating characteristic; HBV, hepatitis B virus.
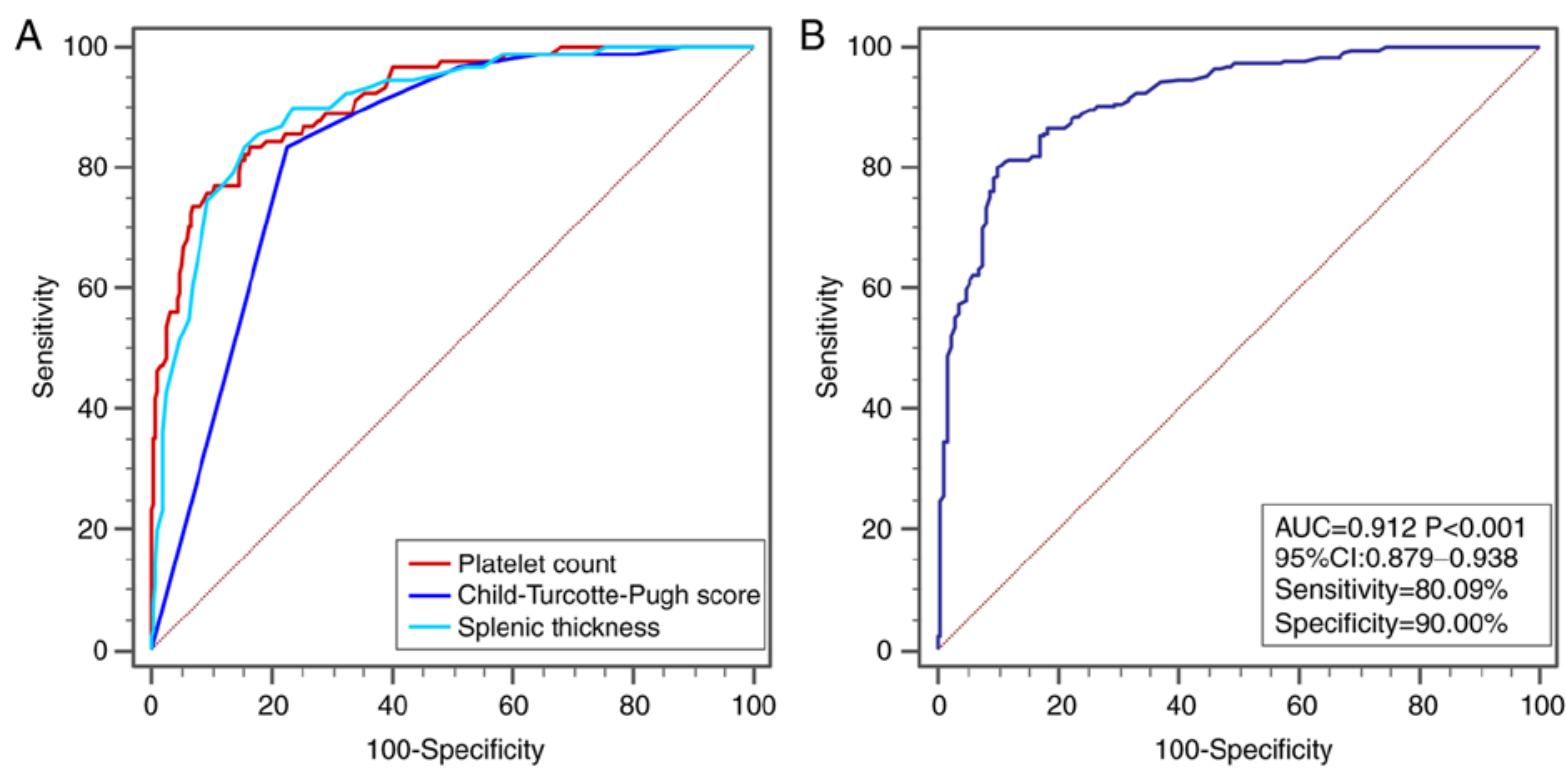

Figure 3. The discriminative ability of platelet count for early-stage cirrhosis. (A) Comparison of ROC curves for the use of platelet count, Child-Turcotte-Pugh score and splenic thickness to identify cirrhosis; (B) ROC curve of platelet count for diagnosing compensated cirrhosis. AUC, area under the ROC curve; ROC, receiver operating characteristic.

Over the past decades, certain studies have attempted to demonstrate that certain non-invasive indicators are able to distinguish liver fibrosis, liver inflammation or liver function $(22,23)$. Furthermore, the platelet count was able to indicate the degree of fibrosis in non-alcoholic fatty liver disease $(10,11)$, and the platelet count was associated with the degree of hepatic fibrosis in chronic hepatitis $\mathrm{C}$ via the mRNA expression of platelet-derived growth factor A (24). An animal study also suggested that platelets produce platelet-derived growth factor-b to activate hepatic stellate cells and promote fibrosis (25). Thus, these studies demonstrated that the platelet count was closely linked to liver function and liver fibrosis.
In the present study, the platelet count was significantly lower in patients with chronic HBV infection and CTP-B/C, and inversely correlated with the CTP score. In particular, the platelet count was found to be independently associated with CTP-B/C and used to distinguish CTP-B/C with an AUC of 0.791 , optimal cut-off value of $73 \times 10^{9} / 1$, sensitivity of $70.73 \%$ and specificity of $75.18 \%$ in patients with normal ALT levels. Therefore, platelets were confirmed to be linked to the severity of liver function in patients with chronic HBV infection. Another study suggested that the increase of platelets was associated with the improvement of liver fibrosis in patients with CHB with significant fibrosis 
Table III. Baseline characteristics of patients who underwent liver biopsy.

\begin{tabular}{|c|c|c|c|}
\hline Variable & $\leq \mathrm{F} 3(\mathrm{n}=6)$ & $\mathrm{F} 4(\mathrm{n}=47)$ & P-value \\
\hline Age (years) & $47.83 \pm 5.15$ & $50.45 \pm 8.88$ & 0.486 \\
\hline Male sex & $5(83.33)$ & 35 (74.47) & 0.635 \\
\hline Platelet count $\left(\times 10^{9} / 1\right)$ & $152.83 \pm 58.78$ & $103.15 \pm 42.97$ & 0.013 \\
\hline $\operatorname{AST}(\mathrm{U} / \mathrm{l})$ & $40.67 \pm 22.93$ & $44.11 \pm 26.71$ & 0.764 \\
\hline $\operatorname{ALT}(\mathrm{U} / \mathrm{l})$ & $59.50 \pm 39.07$ & $41.15 \pm 23.94$ & 0.107 \\
\hline $\mathrm{TP}(\mathrm{g} / \mathrm{l})$ & $74.66 \pm 5.08$ & $70.10 \pm 7.62$ & 0.162 \\
\hline Albumin (g/l) & $47.10 \pm 4.52$ & $42.88 \pm 4.26$ & 0.027 \\
\hline $\mathrm{TBIL}(\mu \mathrm{mol} / \mathrm{l})$ & $20.08 \pm 8.69$ & $21.32 \pm 12.17$ & 0.902 \\
\hline DBIL $(\mu \mathrm{mol} / \mathrm{l})$ & $3.77 \pm 1.56$ & $5.87 \pm 7.41$ & 0.207 \\
\hline IBIL $(\mu \mathrm{mol} / \mathrm{l})$ & $16.32 \pm 7.16$ & $15.45 \pm 6.41$ & 0.758 \\
\hline CHE (KU/l) & $8.05 \pm 1.22$ & $5.85 \pm 1.55$ & 0.002 \\
\hline $\mathrm{TC}(\mathrm{mmol} / \mathrm{l})$ & $4.40 \pm 0.73$ & $3.58 \pm 0.76$ & 0.016 \\
\hline $\mathrm{PT}(\mathrm{sec})$ & $11.53 \pm 1.18$ & $12.91 \pm 1.62$ & 0.049 \\
\hline PTA $(\%)$ & $99.18 \pm 19.54$ & $81.87 \pm 13.43$ & 0.007 \\
\hline $\mathrm{APTT}(\mathrm{sec})$ & $34.22 \pm 6.70$ & $33.69 \pm 5.91$ & 0.840 \\
\hline Splenic thickness $(\mathrm{n}, \mathrm{mm})$ & $2,38.50 \pm 7.78$ & $19,47.47 \pm 8.00$ & 0.147 \\
\hline Portal vein diameter $(\mathrm{n}, \mathrm{mm})$ & $5,10.80 \pm 1.48$ & $24,11.79 \pm 1.32$ & 0.145 \\
\hline
\end{tabular}

Values are expressed as the mean \pm standard deviation or $\mathrm{n}(\%)$. ALT, alanine aminotransferase; AST, aspartate aminotransferase; TBIL, total bilirubin; DBIL, direct bilirubin; IBIL, indirect bilirubin; TP, total protein; CHE, cholinesterase; TC, total cholesterol; PT, prothrombin time; PTA, prothrombin activity; APTT, activated partial thromboplastin time.

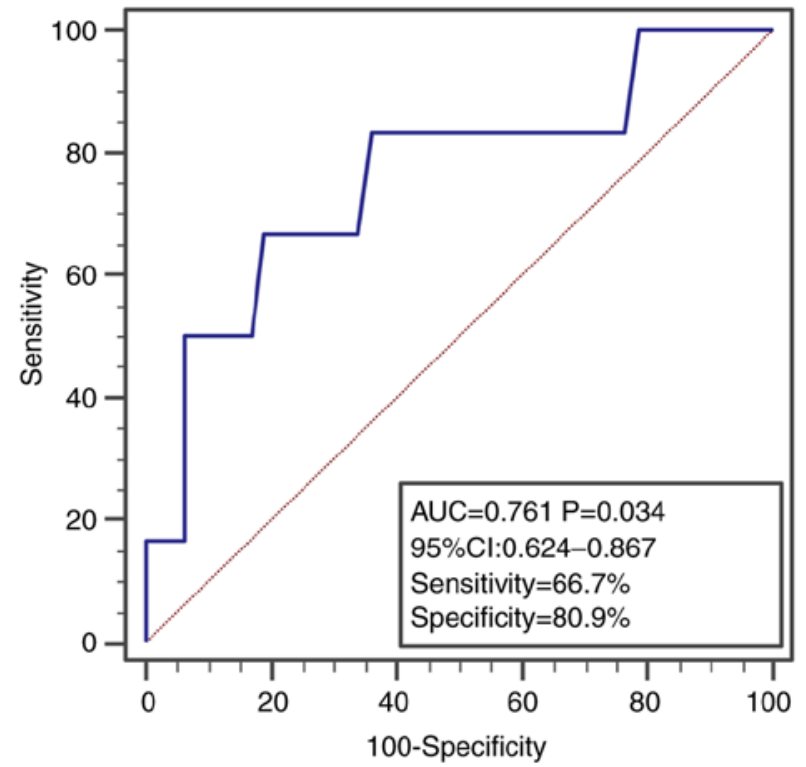

Figure 4. ROC of the platelet count for identifying pathological stage F4 in patients with chronic hepatitis B infection who underwent liver biopsy. AUC, area under the ROC curve; ROC, receiver operating characteristic.

receiving anti-viral therapy (8). This also indirectly indicated the close association between platelets and fibrosis in patients with hepatitis B.

The stage of compensated cirrhosis in CHB is difficult to distinguish without performing an invasive examination. Furthermore, compensated cirrhosis is associated with an increased risk of developing varices, overt
Table IV. Correlation between variables and the pathological stage.

\begin{tabular}{lrc}
\hline Variable & r-value & P-value \\
\hline Platelet count & -0.295 & 0.032 \\
Albumin & -0.279 & 0.043 \\
CHE & -0.392 & 0.004 \\
PT & 0.334 & 0.014 \\
PTA & -0.328 & 0.016
\end{tabular}

CHE, cholinesterase; PT, prothrombin time; PTA, prothrombin activity.

Table V. Independently associated indicators for cirrhosis (pathological stage IV) obtained by multivariate analysis of the indicators with statistical differences in Table III.

\begin{tabular}{lccc}
\hline Variable & OR & $95 \%$ CI & P-value \\
\hline Platelet count & 0.978 & $0.960-0.997$ & 0.026 \\
ALT & 0.980 & $0.950-1.010$ & 0.170 \\
Age & 1.034 & $0.913-1.171$ & 0.425 \\
\hline
\end{tabular}

ALT, alanine aminotransferase; OR, odds ratio; CI, confidence interval.

clinical decompensation (ascites, variceal hemorrhage, hepatic encephalopathy), post-surgical decompensation and 
hepatocellular carcinoma (26). Therefore, the present study focused on the association between platelets and cirrhosis, particularly compensated cirrhosis. The results indicated that the platelet count was inversely correlated with FIB-4 and APRI, which are a non-invasive diagnostic model for hepatic fibrosis. The platelet count was independently associated with cirrhosis. The AUC for the platelet count to indicate cirrhosis (0.927) was significantly higher than that for splenic thickness and CTP score. For early-stage cirrhosis, the platelet count had the best discriminative ability in distinguishing compensated cirrhosis. The AUC was 0.912 with an optimal cut-off value of $117 \times 10^{9} / 1$, sensitivity of $80.09 \%$ and specificity of $90.00 \%$. Therefore, it may be speculated that patients with chronic HBV infection with platelet counts of $<117 \times 10^{9} / 1$ were likely to already have early-stage cirrhosis based on the results.

In addition, the liver biopsy results were used to confirm the above-mentioned association. The results indicated that the platelet count was inversely correlated with the histological stage of fibrosis and independently associated with F4. ROC curve analysis demonstrated that the platelet count is an effective diagnostic indicator for the F4 stage with an AUC of 0.761 . Therefore, the present study further confirmed that the platelet count is closely associated with histological severity in patients with chronic HBV infection.

Of note, the present study had certain limitations. First, no basic experiments were performed to confirm the relevant mechanisms. Additionally, the predictive value of the platelet count to assess the severity of liver injury was not proven. However, based on the rigorous research design and associated results, it was possible to draw conclusions.

In conclusion, the present study confirmed for the first time that the platelet count is independently associated with moderate to severe liver function impairment and cirrhosis in patients with chronic HBV infection. The platelet count may be used to determine the severity of liver injury and liver fibrosis.

\section{Acknowledgements}

Not applicable.

\section{Funding}

The present study was financially supported by the National Natural Science Foundation (grant no. 81800528), the Hospital Fund from the First Hospital of Lanzhou University (grant no. ldyyyn2017-17), the Gansu Health Industry Research Project (grant no. GSWSKY2018-24), the Science and Technology Development Project of Chengguan District (grant no. 2018SHFZ0023), the National Science and Technology Key Project (grant nos. 2017ZX10201201, 2017ZX10203201-005, 2017ZX10202203-006-001 and 2017ZX10302201-004-002) and the Beijing Municipal Administration of Hospital's Ascent Plan (grant no. DFL20151601).

\section{Availability of data and materials}

The datasets used and/or analyzed during the present study are available from the corresponding author on reasonable request.

\section{Authors' contributions}

JFL and ZPD designed the study; YTY, LTY, LLW, WZ and YC collated the data; YTY, LTZ, SJZ and QFC analyzed the data; YTY, LLW and ZPD wrote the paper; JFL, YC, SJZ and $Z P D$ revised the paper. All authors approved the final version of the manuscript.

\section{Ethics approval and consent to participate}

The current study meets the ethical requirements of the Ethics Committee of the First Hospital of Lanzhou University (Lanzhou, China) and received approval. Patients were not required to give informed consent, as the analysis used anonymous clinical data obtained after each patient agreed to treatment by written informed consent.

\section{Patient consent for publication}

Not applicable.

\section{Competing interests}

The authors declare that they have no competing interests.

\section{References}

1. World Health Organization: In: Guidelines for the prevention, care and treatment of persons with chronic hepatitis B infection. Geneva, World Health Organization, 2015.

2. Kurokawa T, Zheng YW and Ohkohchi N: Novel functions of platelets in the liver. J Gastroenterol Hepatol 31: 745-751, 2016.

3. Minamino T, Ito Y, Ohkubo H, Shimuzu Y, Kojo K, Nishizwa N, Amano H, Narumiya S, Koizumi W and Majima M: Adhesion of platelets through thromboxane $\mathrm{A}(2)$ receptor signaling facilitates liver repair during acute chemical-induced hepatotoxicity. Life Sci 132: 85-92, 2015.

4. Meyer J, Lejmi E, Fontana P, Morel P, Gonelle-Gispert C and Bühler L: A focus on the role of platelets in liver regeneration: Do platelet-endothelial cell interactions initiate the regenerative process? J Hepatol 63: 1263-1271, 2015.

5. Nowatari T, Murata S, Fukunaga K and Ohkohchi N: Role of platelets in chronic liver disease and acute liver injury. Hepatol Res 44: 165-172, 2014

6. Afdhal N, McHutchison J, Brown R, Jacobson I, Manns M, Poordad F, Weksler B and Esteban R: Thrombocytopenia associated with chronic liver disease. J Hepatol 48: 1000-1007, 2008.

7. Peck-Radosavljevic M: Thrombocytopenia in liver disease. Can J Gastroenterol 14 (Suppl D): 60D-66D, 2000.

8. Wang L, Wang B, You H, Wu X, Zhou J, Ou X and Jia J: Platelets' increase is associated with improvement of liver fibrosis in entecavir-treated chronic hepatitis B patients with significant liver fibrosis. Hepatol Int 12: 237-243, 2018.

9. Myers RP, De Torres M, Imbert-Bismut F, Ratziu V, Charlotte F and Poynard T; MULTIVIRC Group: Biochemical markers of fibrosis in patients with chronic hepatitis C: A comparison with prothrombin time, platelet count, and age-platelet index. Dig Dis Sci 48: 146-153, 2003.

10. Milovanovic Alempijevic T, Stojkovic Lalosevic M, Dumic I, Jocic N, Pavlovic Markovic A, Dragasevic S, Jovicic I, Lukic S, Popovic D and Milosavljevic T: Diagnostic accuracy of platelet count and platelet indices in noninvasive assessment of fibrosis in nonalcoholic fatty liver disease patients. Can J Gastroenterol Hepatol 2017: 6070135, 2017.

11. Yoneda M, Fujii H, Sumida Y, Hyogo H, Itoh Y, Ono M, Eguchi Y, Suzuki Y, Aoki N, Kanemasa K, et al: Platelet count for predicting fibrosis in nonalcoholic fatty liver disease. J Gastroenterol 46: 1300-1306, 2011.

12. Garjani A, Safaeiyan A and Khoshbaten M: Association between platelet count as a noninvasive marker and ultrasonographic grading in patients with nonalcoholic Fatty liver disease. Hepat Mon 15: e24449, 2015. 
13. Garcia-Tsao G, Abraldes JG, Berzigotti A and Bosch J: Portal hypertensive bleeding in cirrhosis: Risk stratification, diagnosis, and management: 2016 practice guidance by the American Association for the study of liver diseases. Hepatology 65: 310-335, 2017

14. Bedossa P and Poynard T: An algorithm for the grading of activity in chronic hepatitis $C$. The METAVIR cooperative study group. Hepatology 24: 289-293, 1996.

15. Wai CT, Greenson JK, Fontana RJ, Kalbfleisch JD, Marrero JA, Conjeevaram HS and Lok AS: A simple noninvasive index can predict both significant fibrosis and cirrhosis in patients with chronic hepatitis C. Hepatology 38: 518-526, 2003.

16. Sterling RK, Lissen E, Clumeck N, Sola R, Correa MC, Montaner J, S Sulkowski M, Torriani FJ, Dieterich DT, Thomas DL, et al: Development of a simple noninvasive index to predict significant fibrosis in patients with $\mathrm{HIV} / \mathrm{HCV}$ coinfection. Hepatology 43: 1317-1325, 2006.

17. Samiullah S, Qasim R, Khalid S, Hussain BG, Mukhtair J and Akbar Y: Evaluation of creatinine-modified child pugh score for predicting short-term prognosis of patients with decompensated cirrhosis of liver as compare to original child pugh score. J Ayub Med Coll Abbottabad 21: 64-67, 2009.

18. Khokhar N: Serum aminotransferase levels and platelet count as predictive factor of fibrosis and cirrhosis in patients with chronic hepatitis C infection. J Pak Med Assoc 53: 101-104, 2003.

19. Hsu CW, Liang KH, Lin CL, Wang TH and Yeh CT: Platelet counts modulate the quantitative relationship between hepatitis B viral DNA and surface antigen concentrations: A cross-sectional study of hematological, histological and viral factors. BMC Infect Dis 17: 9, 2017

20. Seto WK, Wong DK, Fung J, Ip PP, Yuen JC, Hung IF, Lai CL and Yuen MF: High hepatitis B surface antigen levels predict insignificant fibrosis in hepatitis $\mathrm{B}$ e antigen positive chronic hepatitis B. PLoS One 7: e43087, 2012.
21. Pan Y, Muheremu A, Wu X and Liu J: Relationship between platelet parameters and hepatic pathology in patients with chronic hepatitis B infection-a retrospective cohort study of 677 patients. J Int Med Res 44: 779-786, 2016.

22. Liu DP, Lu W, Zhang ZQ, Wang YB, Ding RR, Zhou XL, Huang D and Li XF: Comparative evaluation of GPR versus APRI and FIB-4 in predicting different levels of liver fibrosis of chronic hepatitis B. J Viral Hepat 25: 581-589, 2018.

23. Li Q, Lu C, Li W, Huang Y and Chen L: Globulin-platelet model predicts significant fibrosis and cirrhosis in CHB patients with high HBV DNA and mildly elevated alanine transaminase levels. Clin Exp Med 18: 71-78, 2018.

24. Tanikawa AA, Grotto RM, Silva GF, Ferrasi AC, Sarnighausen VC and Pardini MI: Platelet-derived growth factor A mRNA in platelets is associated with the degree of hepatic fibrosis in chronic hepatitis C. Rev Soc Bras Med Trop 50: 113-116, 2017.

25. Yoshida S, Ikenaga N, Liu SB, Peng ZW, Chung J, Sverdlov DY, Miyamoto M, Kim YO, Ogawa S, Arch RH, et al: Extrahepatic platelet-derived growth factor- $\beta$, delivered by platelets, promotes activation of hepatic stellate cells and biliary fibrosis in mice. Gastroenterology 147: 1378-1392, 2014.

26. de Franchis R; Baveno VI Faculty: Expanding consensus in portal hypertension: Report of the Baveno VI consensus workshop: Stratifying risk and individualizing care for portal hypertension. J Hepatol 63: 743-752, 2015.

This work is licensed under a Creative Commons Attribution-NonCommercial-NoDerivatives 4.0 International (CC BY-NC-ND 4.0) License. 